\title{
Computational modelling of cathodic protection systems for pipelines in multi-layer soil
}

\author{
A. B. Peratta, J. M. W. Baynham \& R. A. Adey \\ CM BEASY Ltd, UK
}

\begin{abstract}
Computational modelling of cathodic protection (CP) systems involving thin multi-layer media represents a real challenge in terms of accuracy and efficiency required in the numerical calculation. In the case of $\mathrm{CP}$ for transmission pipelines, these long metallic structures are usually buried a distance $\mathrm{H}$ (approximately a metre or so) below ground level and extend horizontally typically more than ten thousand times H. A number of impressed current anode beds are distributed along the pipeline, providing protection against corrosion of the structure. In addition, the vertically stratified nature of the soil needs to be considered in the model, in order to obtain more accurate representation of the environment. This is particularly relevant when considering the effect of different types of rocks, soil porosity, or water saturation, at different depths. This type of scenario requires three dimensional modelling involving a thin multi layered electrolyte, with a typical aspect ratio (lateral extension to thickness) of the order 1E4 to 1E6. The paper presents an efficient and accurate computational approach based on the Boundary Element Method for simulating the level of protection against corrosion of the pipeline as well as current densities and electric potential in different points of the soil. The resulting modelling approach is then applied to assessing real case scenarios. The simulation approach considers the non linear electrode kinetics on the metal surfaces in the form of polarisation data and also the internal resistance of the pipeline and other electrical connections involved in the CP system. Example applications are presented showing how the model can be used to predict the "signatures" associated with different defect types in the pipe coating.
\end{abstract}

Keywords: cathodic protection, multi-layer, Boundary Element Method, transmission pipelines. 


\section{Introduction}

One of the most effective ways to control corrosion in metallic structures embedded in an electrolyte, such as the case of underground or undersea transmission pipelines, is by means of a combination of coating and impressed current cathodic protection (ICCP) systems. Field surveys such as the Direct Current Voltage Gradient (DCVG), and monitoring systems are aimed at different aspects of the assessment of the corrosion control system. Field measurements require skilled technicians, are often expensive and difficult to obtain. In addition, different sections of the pipeline are not always accessible for the surveyors, and in most cases the noise in the potential measurements can mask developing defects in the pipeline coating. Moreover, the interpretation of field data and its correlation to the performance level of the corrosion control system is not always straightforward.

The direct computational modelling of CP systems offers a variety of tools for processing the information collected from the field and complementing it, allowing better interpretation of field measurements, as well as enabling the correlation against the $\mathrm{CP}$ design parameters and their impact on the observable magnitudes.

By direct CP simulation we mean the process of predicting field results such as:

- $\quad$ ON and OFF potentials,

- $\quad$ potential gradients and currents fields in the soil, as well as

- $\quad$ over potential and current density along the pipeline,

Provided that the ICCP design parameters such as for example:

- geometrical arrangement and material properties of pipelines and anodes,

- $\quad$ electrical resistivity distribution of the soil,

- type and location of the electrical connections between rectifiers, anodes and structure

- Coating breakdown factor along the pipeline

are given as input data.

The direct simulation of ICCP systems by computational modelling has achieved in the past few years quite a mature state producing reliable results for industrial environments. This type of simulation is particularly useful at the design stage for the analysis of "what-if" case scenarios including the problems of interference with foreign CP systems or metallic structures.

The purpose of this work is to present an efficient 3D direct simulation tool based on the Boundary Element Method, applied to two case scenarios involving transmission pipelines. The paper is focused on the effects in the potential measurements introduced by variable breakdown factors associated with the coating.

This document is organised in the following way. Section 2 describes modelling approach and provides pointers to the relevant literature involved in the method. Section 3 introduces a simple case for study where the effects of localised damages in the coating along the pipeline can be detected. Section 4 
shows a more realistic case scenario involving three parallel pipelines running along the same corridor. Finally Section 5 establishes the conclusions.

\section{Direct modelling}

The direct modelling consists of obtaining results of electric potential and current density any point in the electrolyte and at surfaces of electrodes. Under most common situations, this requires solving the steady state charge conservation equation in the electrolyte in $3 \mathrm{D}$ space given by:

$$
\nabla \cdot \mathbf{j}=0, \quad \mathbf{x} \in \Omega
$$

where

$$
\mathbf{j}=-\sigma(\mathbf{x}) \nabla u(\mathbf{x})
$$

represents current density, $\sigma$ is the electrolyte conductivity, $\mathrm{u}(\mathrm{x})$ is the potential field, $\nabla$ is the 3D Laplace operator, and $\Omega$ represents the integration domain (electrolyte).

Eqs. (1)-(2) can be solved together with the corresponding boundary conditions which are usually prescribed by imposing polarisation curves at the electrode surfaces, isolating conditions at ground level, and/or fixed potentials at any known equipotential surfaces in the electrolyte (if any).

The Boundary Element Method (BEM) [1] has been widely used to solve Laplacian equations and in particular simulate cathodic protection systems for underground and offshore structures [2-4]. The most significant advantages of the method are first that the formulation is based on the fundamental solution of the leading partial differential operator in the governing equation, and second that it requires only mesh discretisation on the boundaries of the problem. The former aspect confers high accuracy, while the latter substantially simplifies the pre-processing stage of the model, since volume discretisation is not needed.

The forward modelling of long transmission pipelines involves considering the soil as a thin film electrolyte (see Figure 2), since the pipeline span (L) is much bigger than the soil depth relevant for the modelling (h). In addition, the soil is generally stratified in one or many layers along the vertical $(\mathrm{z})$ direction. This thin film stratified integration domain is very difficult to solve with standard modelling techniques such as FEM or BEM. Therefore, in order to be able to solve this type of integration domains without the need for extraordinarily high computational resources, a "multi-layer" BEM has been developed (MLBEM).

The idea behind the ML-BEM is that the stratified nature of the medium is packaged into the corresponding Green's function. In other words, the BEM is applied in the same way as in the case of the homogeneous electrolyte, except that the Green's function for the homogeneous Laplace equation given by $1 /(4 \pi r)$ is replaced by the multi-layer Green's function given by:

$$
G\left(\mathbf{x}_{i}, \mathbf{x}_{j}, m, n\right)=\frac{1}{4 \pi \sigma_{m}} \sum_{k=1}^{N \exp } \frac{\alpha_{i j m l}}{\left\|\mathbf{x}_{i}-\mathbf{x}_{j}+\mathbf{g}_{i j}\right\|}
$$


where $\mathbf{x}$ denotes the $3 \mathrm{D}$ coordinates, the sub indices $\mathbf{i}$ and $\mathbf{j}$ stand for the source and field point, respectively; $m$ and 1 indicate the layer of the source and field points, respectively; $\alpha_{i j m l}$ is a weight coefficient and $\mathbf{g}_{i j m l}$ denotes a displacement vector. The Green's function written in this way can be regarded as the one produced by a weighted method of images.

The calculation of the weight and displacement vectors goes beyond the scope of this paper and can be derived from references [5] and references therein. Finally, the Green's function (3) replaces the 1/r kernel used for homogeneous regions, and the same BEM strategy can be employed.

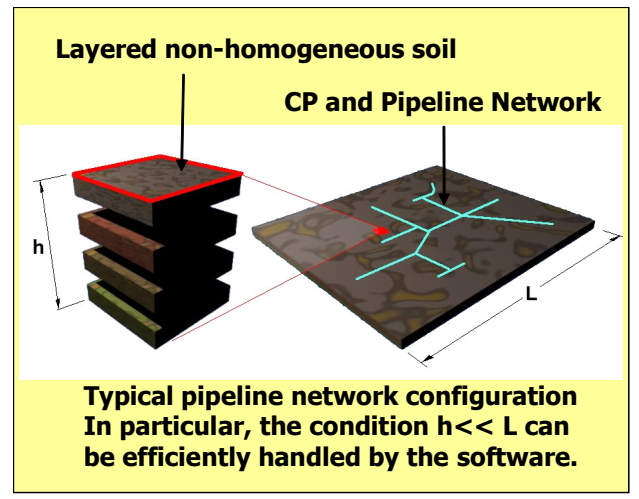

Figure 1: The soil (electrolyte) considered as thin film stratified media.

\section{Case study 1}

Figure 2 shows a $16 \mathrm{~km}$ length of pipeline, composed of 5 electrically connected subsections. At the connection joints between subsections there are four rectifiers delivering constant current to 4 anodes A1 to A4. The table on the right hand side of the figure shows the xy coordinates of the anode in the ground. In the figure, Dz represents the vertical dimension of the anode, which extends down from the ground surface. Details of the pipeline are shown in Table 1: Pipeline specifications. The total current delivered by the anodes is $40 \mathrm{Amps}(10 \mathrm{~A} \mathrm{each})$.

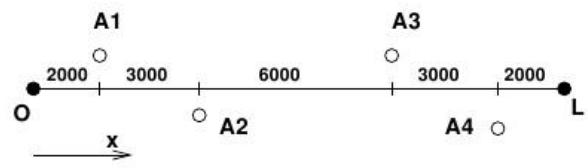

\begin{tabular}{|crccc|}
\hline Anodes & \multicolumn{1}{c}{ x } & y & Dz & diam \\
\hline A1 & 2000 & -200 & $0-50$ & 0.2 \\
A2 & 5000 & +200 & $0-50$ & 0.2 \\
A3 & 11000 & -200 & $0-50$ & 0.2 \\
A4 & 14000 & +200 & $0-50$ & 0.2 \\
\hline
\end{tabular}

Figure 2: $\quad$ Pipeline layout and relevant anode parameters (right).

The soil model consists of two layers, with depths and conductivity as shown in Table 2. The top of the first layer and the bottom of the second layer are insulating. The system of coordinates is such that the pipeline is oriented in $\mathrm{x}$ direction from $\mathrm{x}=0$ to $\mathrm{x}=16000 \mathrm{~m}$; the $\mathrm{z}$ direction indicated depth $\mathrm{z}=0$ represents 
Table 1: $\quad$ Pipeline specifications.

\begin{tabular}{|l|l|l|}
\hline \multicolumn{2}{|c|}{ PIPELINE } \\
\hline $\mathrm{L}$ & $16 \mathrm{~km}$ & Length \\
$\mathrm{z}$ & $4 \mathrm{~m}$ & Depth \\
$D_{e}$ & $1 \mathrm{~m}$ & External diameter \\
$t$ & $0.01 \mathrm{~m}$ & Wall thickness \\
$\sigma_{s}$ & $5.74713 \times 10^{6} \mathrm{~S} / \mathrm{m}$ & Steel conductivity \\
$\rho$ & $5.59453 \times 10^{-6} \Omega / m$ & Resistance per unit lenght \\
\hline
\end{tabular}

Table 2: $\quad$ Soil properties.

\begin{tabular}{|l|l|l|}
\hline Layer & Zmin to Zmax & Conductivity $[\mathrm{S} / \mathrm{m}]$ \\
\hline 1 & 0 to $-30 \mathrm{~m}$ & 0.02 \\
\hline 2 & $-30 \mathrm{~m}$ to $-100 \mathrm{~m}$ & 0.007 \\
\hline
\end{tabular}

ground level, and $\mathrm{z}=4 \mathrm{~m}$ is the centreline of the pipeline). Note that models with this type of boundary conditions are not easily solved with standard techniques such as half space, or method of images [6].

Figure 3 shows the over potential along the pipeline (which is almost the same as the OFF potential calculated above the pipeline at ground level) for different conditions of the pipeline. The red curve corresponds to a uniform breakdown factor $\mathrm{BF} 0=1(100 \%$, bare steel $)$ for the whole pipeline. The green curve corresponds to the case of a uniformly partially damaged coating with BF0 $=0.02(2 \%)$. The black dotted curve corresponds to undamaged uniform coating characterised by uniform BF0 $=0.01(1 \%)$. The Breakdown Factor (BF) is a convenient method for representing general degradation of the pipeline coating. A $10 \%$ BF implies that the coating has degraded to the point where an area equivalent to $10 \%$ of the surface area is bare metal.

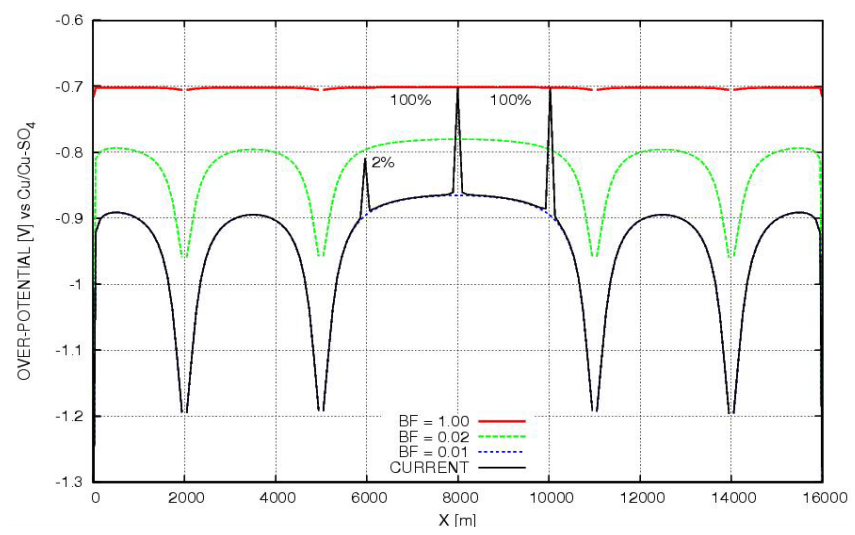

Figure 3: Comparison between different coating damages in the over potential distribution along the pipeline.

The solid black curve shows the pattern of results produced by 3 localised coating defects at 6000,8000 and $1000 \mathrm{~m}$ (with local breakdown factor BF equal 
to $0.02(2 \%), 1(100 \%)$ and $1(100 \%)$, respectively) on the pipeline which has an otherwise uniform breakdown factor of $1 \%$. The length of each defect along the pipeline is $2 \mathrm{~m}$. It can be observed that the effect of the local damage on the over potential is very localised, and that there is no observable general shift of overpotential. This is because in this case the change of current density is small at regions away from the damage.

The pattern observed for the case of 19 localised defects is shown in Figure 4. The distribution of defects is shown in Figure 5 in terms of the breakdown factor in function of length along the pipeline.

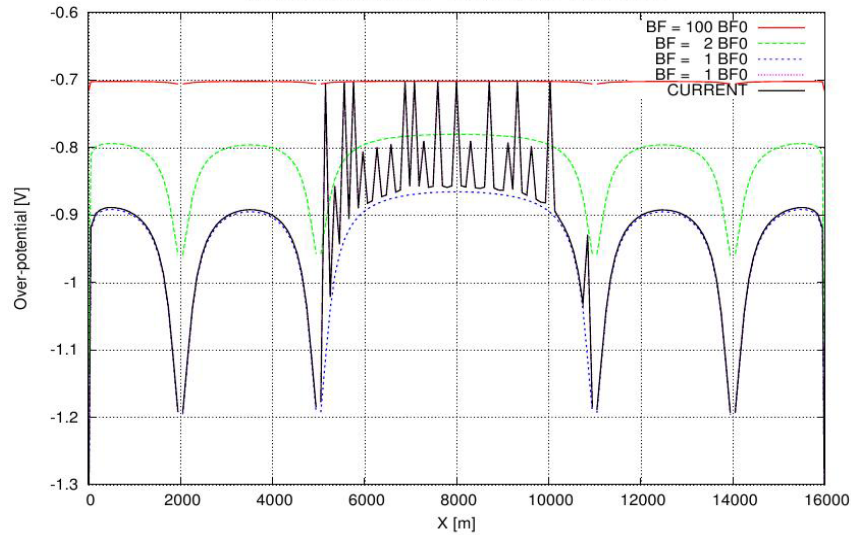

Figure 4: "Signature"Over potential along the pipeline produced by 19 defects distributed as shown in Figure 5.

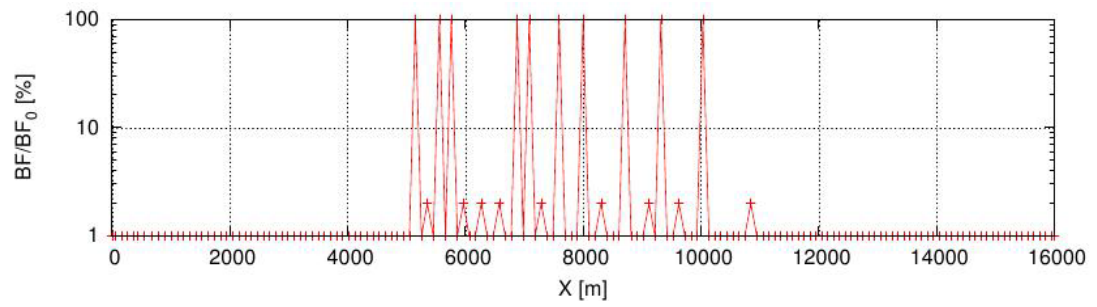

Figure 5: $\quad$ BF along pipeline corresponding to the pattern shown in Figure 4.

The method of using breakdown factors in the model to represent local breakdown in the quality of the coating is adequate when the area of damage is relatively large (i.e. larger than a few metres) and the model is capable of predicting what the potential and gradient signatures generated near the pipeline are. This provides a methodology of characterising the size and extent of the defect from its signature. However for more distinct local holidays in the coating a different type of model is required as it is necessary to model the variation of the field around the pipe as discussed in the next section.

The simulation tool is also capable of modelling in more detail the effect of localised damage, and the pattern/signature of potential and potential gradient at 


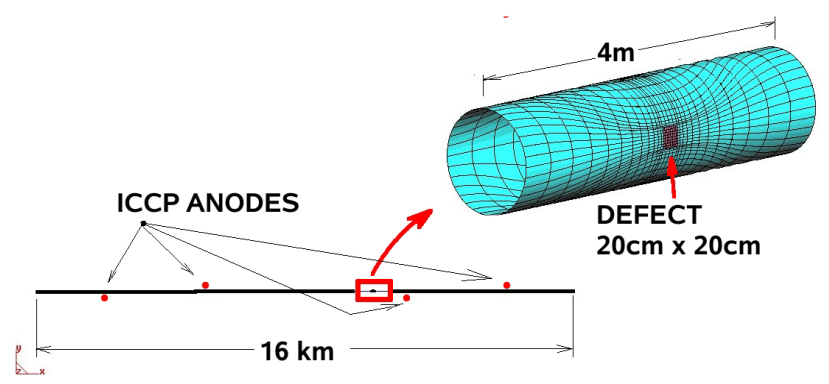

Figure 6: Detailed representation of a local defect.

ground level produced by small defects in the coating. The rest of this sections shows the simulation results applied to the case of a $0.2 \times 0.2 \mathrm{~m}$ zone of damaged coating at $x=10000 \mathrm{~m}$. The situation is illustrated in Figure 6 where a three dimensional model of the pipeline is shown. This model is different to the previous in that the elements consider the variation of the coating quality around the pipe.

Figure 7 shows the over potential near the holiday. The rest of the pipeline (total length $16 \mathrm{~km}$ ) is also included in the model and it is considered to have uniform coating breakdown factor of $0.1 \%$. In this case each ICCP anode injects $2 \mathrm{~A}$ to the system, and an increased number of elements are used in the region that contains holiday.

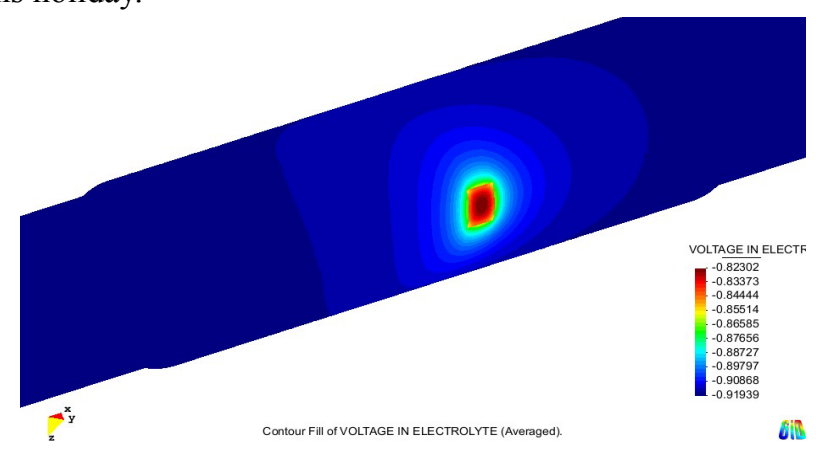

Figure 7: Overpotential near a lateral holiday.

The on-potential at ground level with respect to the pipeline can be observed in Figure 8.

Figure 9 represents the surface distribution of transversal current density at ground level when the CP system is switched on. The observation plane (at ground level) covers a region of $30 \times 15 \mathrm{~m}$ centred at $\mathrm{x}=10000 \mathrm{~m}$.

It is interesting to compare the different types of pattern produced by the different types and location of defects in the pipeline. In particular, the contrast of voltage and voltage gradient profiles with respect to the case of a healthy pipeline (no holiday at all) is shown in Figures 10 and 11.

Figure 10 on the left shows a comparison of longitudinal voltage gradients at ground $(\mathrm{z}=0)$ level at $\mathrm{y}=0.5 \mathrm{~m}$ (The pipeline is in the plane $\mathrm{y}=0$, at depth $\mathrm{z}=$ 
$4 \mathrm{~m}$ ). The figure compares the case of a uniformly coated pipeline (no defect) and the same pipeline with the holiday (defect). Figure 10 on the right shows the same comparison but with the transversal voltage gradient at different $y$ positions.

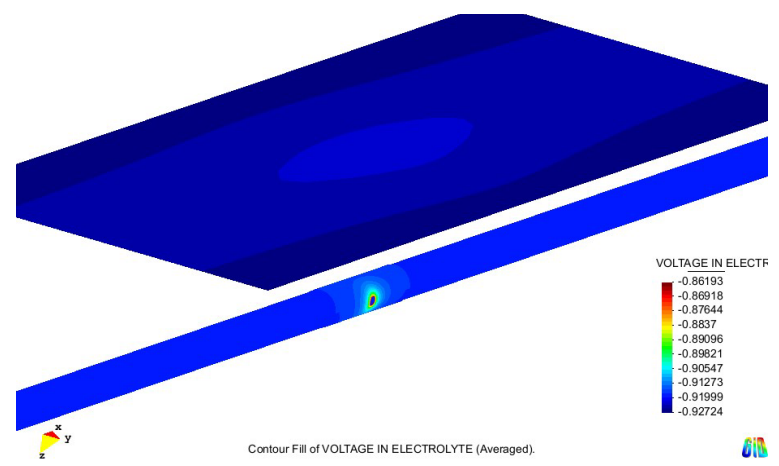

Figure 8: $\quad$ Soil-to-pipe potential at ground level and electrolyte potential on the pipeline.

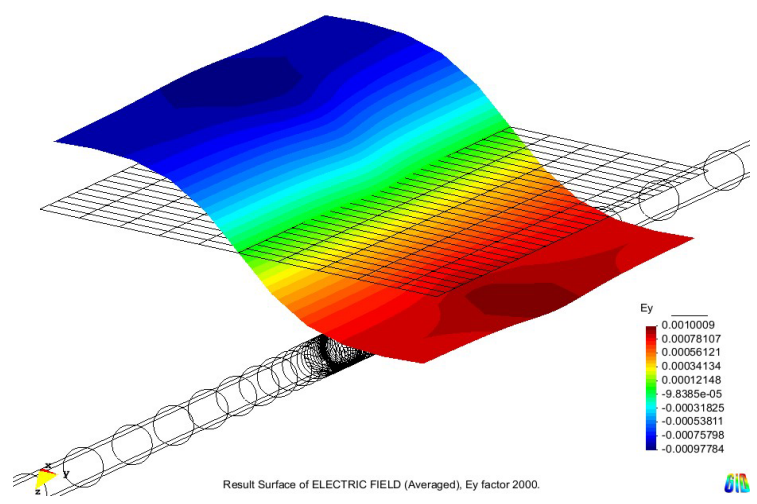

Figure 9: Surface distribution of Y component of current density at ground level.
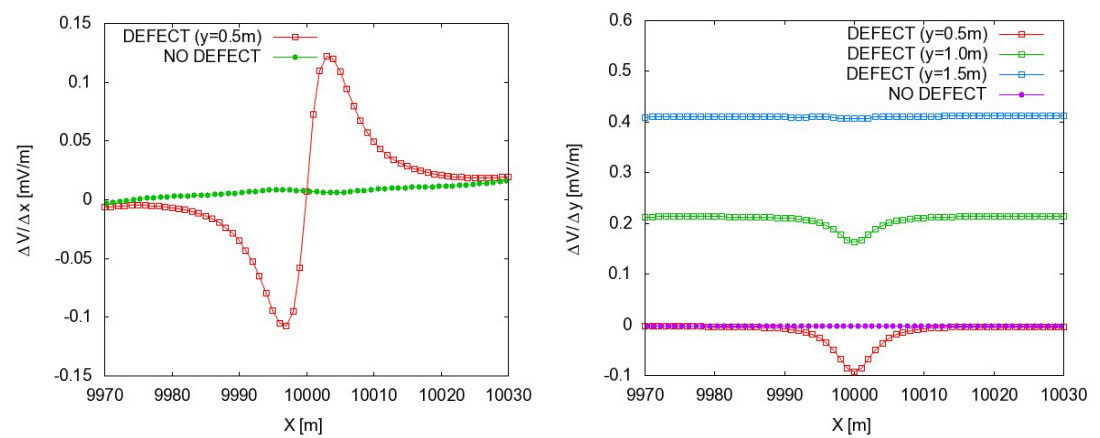

Figure 10: Voltage gradient in X (left) and Y (right) direction at ground level. 
Figure 11 compares the potential profiles for the pipeline with and without holiday. The picture on the left shows the soil-to-pipe ON-potential at ground level above the pipeline (i.e. $\mathrm{y}=0, \mathrm{z}=0$ ). The corresponding over potential profile of each pipeline is shown on the right $(z=4 \mathrm{~m}, \mathrm{y}=0.5 \mathrm{~m})$.

The results demonstrate how the pattern of electrical signatures observed at ground level due to the presence of small defects on the coatings can be determined in addition to the attenuation of the potential and potential gradients as the observation point moves farther away from the coating.
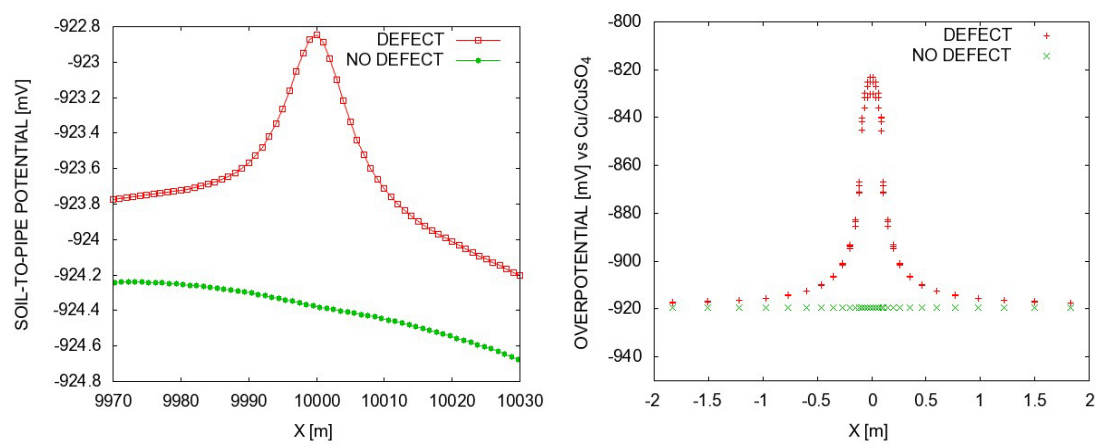

Figure 11: (Left) Soil-to-pipe potential. (Right) Over potential on pipeline near the defect.

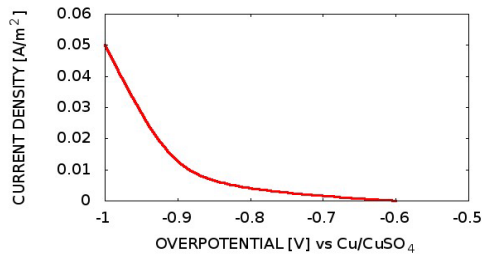

Figure 12: Polarisation curve for the metallic structure.

\section{Case study 2}

The model shown in Figure 13 considers three parallel pipelines of approximately $70 \mathrm{~km}$ in length sharing the same $\mathrm{CP}$ system which consists of 11 ICCP deep anodic beds delivering $10 \mathrm{~A}$ each. The distance between pipelines is $10 \mathrm{~m}$ in the local normal direction. The model considers two soil layers $(0.02 \mathrm{~S} / \mathrm{m}$ from ground level to $-35 \mathrm{~m}$, and 0.005 from $-35 \mathrm{~m}$ to $-100 \mathrm{~m}$ ).

Figure 14 (left) shows the over potential of the three pipelines along their developed length. In this case, the difference between over potential and OFF potential is negligible.

The ohmic drop in the return path due to finite conductivity of the metal is not negligible in large distances, and usually affects the $\mathrm{CP}$ parameters. In this example, the voltage difference at different points of the structure is approximately $300 \mathrm{mV}$, as observed in Figure 14 (right). The maximum 

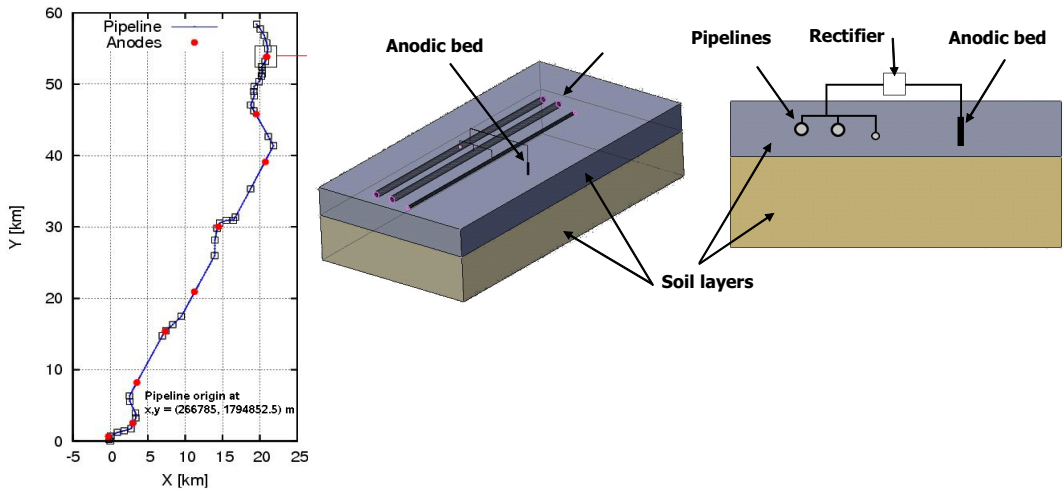

Figure 13: Schematic representation of case study 2. (Left) Plan view, the dots indicate the location of the ICCP anodes. (Middle and right) Detail of electrical connections at each between anode and the pipelines at each connection point.

Table 3: $\quad$ Relevant pipeline properties for case study 2.

\begin{tabular}{|l|l|l|l|l|}
\hline Pipeline Name & & A & B & C \\
\hline Type & & NPS6 SCH80s & NPS16 SCH & NPS10 SCH80 \\
\hline Pipeline length (m) & L & $68442 \mathrm{~m}$ & $68442 \mathrm{~m}$ & $68442 \mathrm{~m}$ \\
\hline Ext.diameter (inch) & De & $6.625 ”$ & $16 ”$ & $10.750 ”$ \\
\hline Wall thickness (Inch) & t & $0.432^{\prime \prime}$ & $0.656 ”$ & $0.594 ”$ \\
\hline Metal resistivity (Ohm m) & rho & $1.74 \mathrm{E}-7$ & $1.74 \mathrm{E}-7$ & $1.74 \mathrm{E}-7$ \\
\hline Breakdown factor & BF & Uniform=50\% & Uniform 3\% & Uniform 100\% \\
\hline
\end{tabular}

Table 4: $\quad$ Anodes location.

\begin{tabular}{|c|c|l|l|l|c|}
\hline Name & $\mathbf{X}$ & \multicolumn{1}{|c|}{$\mathbf{Y}$} & \multicolumn{1}{|c|}{ Name } & $\mathbf{X}$ & $\mathbf{Y}$ \\
\hline A1 & 266543.4 & 1795525.1 & A7 & 285301.0 & 1829520.0 \\
\hline A2 & 269839.0 & 1797394.0 & A8 & 287517.0 & 1833957.0 \\
\hline A3 & 270400.0 & 1803073.0 & A9 & 286274.0 & 1840641.0 \\
\hline A4 & 274181.0 & 1810219.0 & A10 & 287682.0 & 1848656.0 \\
\hline A5 & 278114.0 & 1815776.0 & A11 & 286435.0 & 1853240.0 \\
\hline A6 & 281253.0 & 1824888.0 & & & \\
\hline
\end{tabular}
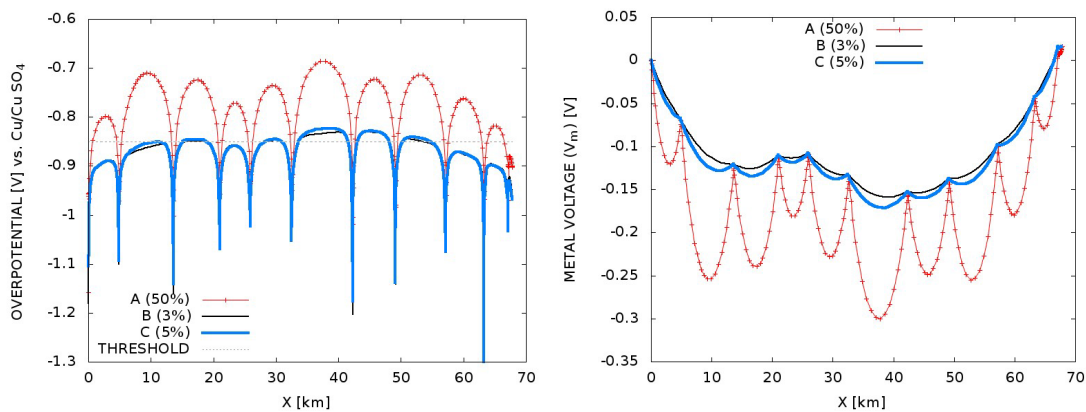

Figure 14: (Left) Over potential along the three pipelines. (Right) Metal voltage potential along the pipelines. 

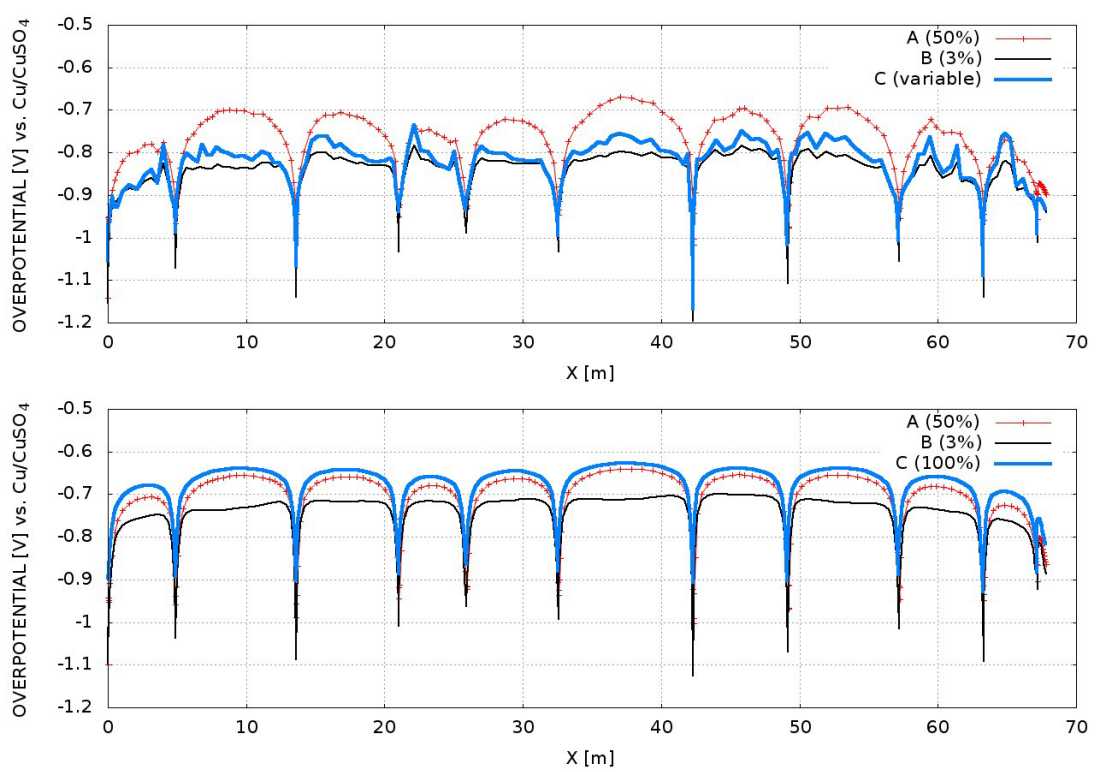

Figure 15: Over potential along pipelines with different coating qualities of pipeline C. (Top) Variable breakdown factor estimated from Close Interval Survey (CIS) measurements. (Bottom) Pipeline $\mathrm{C}$ with $100 \%$ BF.

variations are registered in pipeline A due to its smaller cross sectional area which introduces a higher resistance per unit length.

The simulation has been repeated considering different qualities of the coating for pipeline $C$. The effects of the quality of the coating of pipeline $C$ on the over potential profile along the neighbouring pipelines can be observed in Figure 15. The top figure shows the case of variable breakdown factor estimated from a CIS survey, in this case, the BF ranges from 0 to approximately $35 \%$. The bottom figure represents the case of pipeline $\mathrm{C}$ with no coating at all.

The results obtained demonstrate a number of features. First, the feasibility of the modelling approaches for estimating the quality of the coating using data from survey data which is generally spiky and noisy. Second, they reveal how the quality of the coating of one of the pipelines can affect the level of protection in the neighbouring structures.

\section{Conclusions}

A BEM based modelling tool has been developed for the direct simulation of CP systems involving pipelines in multi-layer media.

The formulation can represent several layers of geological regions in the soil and does not require the mesh discretisation of the interface between layers. As a consequence, the computational cost of the simulation does not increase with the number of layers. 
Two case scenarios were presented in order to illustrate some of the applications of the tool. The first one provides comprehensive results on the impact of the distribution of localised damage to the coating on the over potential from a global point of view. In addition, the effects of a localised coating damage on the local patterns of voltage gradient, soil-to-pipe potential at ground level have been obtained, including both localised degradation and holidays in the coating.

In the second case, the interference between three $70 \mathrm{~km}$ pipelines running parallel along the same corridor has been modelled. The results obtained demonstrate the models ability to represent the case where the damage in the coating of one of the pipelines affects the level of protection of the others.

The modelling tool has been used to investigate cases of interference due to varying quality of coating breakdown factor.

Once calibrated, the simulation becomes a powerful tool for analysing different types of scenarios, to conduct optimisations on existing $\mathrm{CP}$ systems, to provide better interpretation of survey data, and to improve the survey techniques themselves. In particular the modelling approach can be applied to interpret survey data in regions where access is difficult by using adjacent measurements in conjunction with the model to simulate the data required.

\section{References}

[1] C.A. Brebbia, J.C.F. Telles and L.C. Wrobel. Boundary Element Techniques - Theory and Application in Engineering. Springer Verlag Berlin, Heidelberg NY, Tokyo. 1984.

[2] D. P. Riemer and M. E. Orazem, "Modelling Coating Flaws with NonLinear Polarization Curves for Long Pipelines," in Corrosion and Cathodic Protection Modelling and Simulation, Volume 12 of Advances in Boundary Elements, R. A. Adey, editor, WIT press, Southampton, 2005, 225-259.

[3] D. P. Riemer and M. E. Orazem, "Application of Boundary Element Models to Predict the Effectiveness of Coupons for Accessing Cathodic Protection of Buried Structures," Corrosion, 56 (2000) 794-800.

[4] R.A. Adey, J. Baynham. Design and Optimization of Cathodic Protection Systems using Computer Simulation. CORROSION 2000, Paper \} 7 2 3 . Houston, Texas. NACE International, 2000.

[5] Andres B Peratta, John M W Baynham, and Robert A. Adey. A Computational Approach for Assessing Coating Performance in Cathodically Protected Transmission Pipelines. CORROSION 2009, Paper 6595 Atlanta, Georgia. NACE International 2009.

[6] J.D. Jackson. Classical Electrodynamics. 2nd ed. John Wiley \& Sons. NY, Chichester, Brisbane, Toronto, Singapore. 1975 